\title{
Arborescences
}

Revue d'études françaises

\section{Prescriptions institutionnelles et enseignement de l'écriture aux élèves nouvellement arrivés en France}

\section{Marie-Pascale Hamez}

Numéro 1, mars 2011

Identités linguistiques, langues identitaires : à la croisée du prescriptivisme et du patriotisme

URI : https://id.erudit.org/iderudit/1001945ar

DOI : https://doi.org/10.7202/1001945ar

Aller au sommaire du numéro

Éditeur(s)

Département d'études françaises, Université de Toronto

ISSN

1925-5357 (numérique)

Découvrir la revue

Citer cet article

Hamez, M.-P. (2011). Prescriptions institutionnelles et enseignement de l'écriture aux élèves nouvellement arrivés en France. Arborescences, (1), 0-0. https://doi.org/10.7202/1001945ar
Résumé de l'article

L'auteure s'intéresse, dans une optique didactique, à l'enseignement de la langue française en France dans un contexte scolaire particulier : celui des dispositifs d'accueil temporaires destinés aux élèves nouveaux-arrivants non francophones. L'étude examine la manière dont les prescriptions institutionnelles, historiquement situées, ont traité dans leurs configurations successives, de la question de l'entrée dans l'écrit de la langue-culture cible et de l'accès à la littéracie pendant ces quarante dernières années. Organisé selon deux grands axes, l'article s'efforce de préciser les statuts conférés à la diversité linguistique et culturelle des élèves pendant la période étudiée et est fondé sur une analyse des postulats théoriques et didactiques sur lesquels se sont respectivement appuyées les prescriptions.
Tous droits réservés @ Département d'études françaises, Université de Toronto, 2011 document est protégé par la loi sur le droit d'auteur. L’utilisation des services d'Érudit (y compris la reproduction) est assujettie à sa politique d'utilisation que vous pouvez consulter en ligne. 


\title{
PRESCRIPTIONS INSTITUTIONNELLES ET
}

\section{ENSEIGNEMENT DE L'ÉCRITURE AUX ÉLÈVES NOUVELLEMENT ARRIVÉS EN FRANCE}

\author{
Marie-Pascale Hamez \\ Université Charles de Gaulle - Lille 3
}

«La nation fixe comme mission première à l'école de faire partager aux élèves les valeurs de la République. » Article 2 de la Loi du 23 avril 2005 d'orientation et de programme pour l'Avenir de l’École

\section{Introduction}

En France, depuis 2006, c'est le Socle commun de connaissances et de compétences ${ }^{1}$, officiellement appelé « ciment de la Nation » qui reconfigure le système scolaire puisqu'il est érigé en référence pour la rédaction des prescriptions officielles à l'école ${ }^{2}$ et au collège ${ }^{3}$. Le Socle est défini comme un « ensemble de valeurs, de savoirs, de langages et de pratiques dont l'acquisition repose sur la mobilisation de l'École et qui suppose, de la part de l'élève, des efforts et de la persévérance » (Ministère de l’Éducation Nationale $2006: 3$ ).

Par engagement de l'institution, le Socle, production officielle de 30 pages, garantit à tous les savoirs minima en les organisant autour de sept piliers. La maîtrise de la langue française constitue le premier pilier car elle figure «l'outil premier de l'égalité des chances, de la liberté du citoyen et de la civilité : elle permet de communiquer à l'oral comme à l'écrit, dans diverses situations ; elle permet de comprendre et d'exprimer ses droits et ses devoirs ». La priorité accordée à la langue nationale est

\footnotetext{
${ }^{1}$ L'établissement d'un socle commun de connaissances et de compétences est une disposition de la loi d'orientation et de programme pour l'Avenir de l'École du 23 avril 2005. Le décret du 11 juillet 2006 pris en application de la loi organise le contenu du socle autour de sept grands axes, et prend en compte les recommandations du Haut Conseil de l'Éducation (2006). Il s'inscrit en même temps dans la logique des orientations de l'Union européenne en matière d'éducation. Sa mise en œuvre s'échelonne de la rentrée 2007 à la rentrée 2009. Les sept piliers du socle commun sont : la maitrise de la langue française, la pratique d'une langue vivante étrangère, les principaux éléments de mathématiques et la culture scientifique et technologique, la maîtrise des techniques usuelles de l'information et de la communication, la culture humaniste, les compétences sociales et civiques, l'autonomie et l'initiative.

${ }^{2}$ Dans le système éducatif français, il s'agit de l'école primaire qui constitue le premier degré de l'enseignement. Ce degré comprend l'école maternelle (élèves âgés de 2 à 6 ans) et l'école élémentaire (élèves âgés de 6 à 11 ans).

${ }^{3}$ En France, le collège propose le premier cycle des études du second degré. Il accueille des élèves âgés de 11 à 15 ans.
} 
donc toujours fortement liée de manière emblématique à l'idéal républicain. C'est aujourd'hui encore le monolinguisme qui fonde idéologiquement l'approche de la langue dans les programmes de l'Éducation Nationale française. Par ailleurs, d'après le Socle, l’instrument majeur des acquisitions nécessaires à la maîtrise de la langue française n’est autre que «la fréquentation de la littérature française ». C’est pourquoi les plus récents programmes publiés en 2008 pour le collège, recommandent d'articuler des travaux d'écriture en liaison avec l'analyse de textes littéraires, par conséquent d'exposer les élèves à une variété de langue élaborée, fondamentalement abordée dans sa forme écrite et placée par l'institution en situation de norme dominante :

Toute séance d'analyse de textes littéraires comporte avant la séance, pendant, à la fin ou après, des travaux d'écriture. Les activités d'écriture sont variées. Le résumé ou la reformulation d'un texte lu ou d'un propos entendu, l'invention de débuts ou de suites de textes, les insertions, les imitations, les transformations par exemple, sont autant de contrepoints stimulants pour permettre une perception plus fine et plus personnelle des textes. (Ministère de l’Éducation Nationale $2008: 3$ )

Le français enseigné n'est donc pas le français des usages vernaculaires. C'est une variété élaborée, cultivée, fondamentalement abordée dans sa forme écrite sous la forme de conduites langagières complexes. Comment cette variété de français est-elle enseignée aux élèves allophones?

Dans cet article, j'adopte un point de vue de didacticienne pour m’intéresser à l'enseignement de la langue française dans un contexte scolaire particulier: celui des dispositifs d'accueil temporaires destinés aux élèves nouveaux-arrivants non francophones, désignés actuellement par le terme d'ÉNAF : élèves nouvellement arrivés en France. Ces élèves, appelés aussi primo-arrivants, devront au bout d'une année scolaire suivre les mêmes programmes et être confrontés aux mêmes systèmes d'évaluation que les natifs. Ma contribution s'attache en particulier à l'enseignement de l'écriture dans cet environnement sociolinguistique et didactique complexe. Elle étudie la manière dont les prescriptions institutionnelles, historiquement situées, ont traité dans leurs configurations successives, de la question de l'entrée dans l'écrit de la langue-culture cible et de l'accès à la littéracie pendant ces quarante dernières années. Un enjeu fondamental pour tout sujet de l'institution scolaire, spécifiquement pour les élèves allophones puisque c'est surtout l'écrit qui compromet la poursuite de leur scolarité dans les classes dites «ordinaires» ou «banales» du secondaire. (Nonnon 1991)

On sait que les préconisations institutionnelles sont des instruments normatifs qui incarnent la manifestation d'un choix politique à un moment donné de l'histoire d'une nation. Elles s'inscrivent dans une continuité, une tradition culturelle, et ne cessent, comme je le montrerai, d'accumuler et de renouveler des modifications apportées par un groupe social, une société donnée. 
En France, après la Révolution de 1789, les partisans de la République expriment la nécessité de maintenir un pouvoir centralisé. À la fin du XIX ${ }^{\mathrm{e}}$ siècle, les lois Ferry (1881 et 1882) constituent un aboutissement ainsi qu'un instrument de la francisation généralisée et consolident le régime républicain. L'enseignement doit être donné en français, langue de la Révolution, des droits de l'homme et de la République sur tout le territoire. Tout enseignement des langues régionales (par exemple le flamand, le breton, l'auvergnat ou l'arabe et le kabyle en Algérie, annexée en 1848) est interdit.

Après avoir présenté les dispositifs d'accueil actuels, j'articulerai mon étude selon deux grands axes. Le premier axe s'efforce de préciser les statuts conférés à la diversité linguistique et culturelle des élèves pendant la période étudiée. Le deuxième axe est fondé sur une analyse des postulats théoriques et didactiques sur lesquels se sont respectivement appuyées les prescriptions.

\section{Dispositifs d'accueil pour élèves nouvellement arrivés en France}

À l'école, au collège et au lycée, l'institution pratique la double inscription : ces élèves sont d'une part affectés dans une classe correspondant à leur âge où ils suivent des enseignements en éducation physique et sportive, arts plastiques et musique, et d'autre part ils sont regroupés dans une classe d'initiation (CLIN) à l'école élémentaire, ou dans une classe d'accueil (CLA) au collège et au lycée pour un enseignement quotidien de la langue française. Ces regroupements de 15 élèves au maximum atteignent un degré d'hétérogénéité maximale. Les élèves âgés de 11 à 16 ans manifestent des centres d'intérêt différents. Leur langue-culture d'origine varie ainsi que leur niveau de scolarité antérieure, leur degré d'alphabétisation et leur rapport à l'apprentissage du français et dans une plus large extension, à l'École. L'institution recommande que la durée d'accueil dans ces classes spécifiques, variable selon les besoins de chaque élève, n’excède pas un an, l’objectif affiché étant que les élèves puissent suivre, le plus rapidement possible, l'intégralité des enseignements dans une classe du cursus ordinaire. Par ailleurs, dans le second degré, les élèves ayant été très peu ou pas du tout scolarisés dans leurs pays d'origine intègrent une classe d'accueil pour élèves non-scolarisés antérieurement (CLA-NSA). 
Certaines académies ${ }^{4}$ ont également développé des dispositifs de soutien linguistique plus souples: en raison de contraintes géographiques (zones rurales notamment), de la faiblesse des effectifs, ou bien après quelques mois passés en CLIN-CLA, les élèves non-francophones peuvent bénéficier de cours de rattrapage intégrés (CRI) dans les écoles élémentaires ou de modules d'accueil temporaires (MAT) dans les collèges, assurés quelques heures par semaine par un enseignant itinérant. Selon une enquête de la division de l'Évaluation et de la Prospective (M. E. N 2008), les ÉNAF représentaient $0,4 \%$ des effectifs scolaires et étaient majoritairement inscrits dans les académies de Paris, Créteil, Versailles et Lyon. Ce chiffre ne témoigne bien évidemment pas de la réalité du terrain dans la mesure où les dispositifs d'accueil étant majoritairement implantés en milieu urbain, les élèves allophones directement intégrés en classe ordinaire ne sont pas comptabilisés... D’après les données ministérielles, c'est l'Afrique qui reste le continent de provenance le plus fréquemment représenté sauf dans les Départements d'Outre mer où les nouveaux-arrivants sont originaires des Caraïbes. Les prescriptions officielles auxquelles je m’intéresse, médiatisent le travail des enseignants et de leurs élèves au niveau du collège, premier cycle de l'enseignement secondaire. Composantes institutionnelles des pratiques enseignantes, elles présentent un caractère multi-adressé dans la mesure où elles visent non seulement les professeurs, les collectivités territoriales, les parents d'élèves, mais aussi d'après les chiffres les plus récents communiqués par le ministère, 17800 collégiens répartis dans 751 dispositifs d'accueil dont 512 CLA en 2005.

\section{Prescriptivisme, patriotisme et prescriptions institutionnelles}

Les prescriptions institutionnelles actuelles destinées à l'ensemble de la population scolaire s'inscrivent dans une tradition idéologique, politique et culturelle qui fait de la langue de l'école une langue porteuse de l'identité nationale et ce, depuis les lois de Jules Ferry (1881-1882), à une période où la France est un pays plurilingue surtout dans les campagnes et les faubourgs ouvriers des grandes villes. C'est l'époque où les langues régionales transmises par les familles jusqu'en 1930 concurrencent la langue nationale dite «maternelle»: langue celte, langue basque, langues romanes dont l'occitan, langues germaniques... L'école laïque, gratuite et quasi obligatoire diffuse à l'échelle du territoire national la même langue, et devient de ce fait, avec la conscription, l'un des principaux

\footnotetext{
${ }^{4}$ En France, une académie est une circonscription administrative qui relève du ministère de l'Éducation Nationale. C'est l'échelon administratif qui décline en région la politique éducative définie par le gouvernement.
} 
instruments de «nationalisation de la société » par l'État ou encore de formation de l'État-nation français.

Au cours du $\mathrm{XX}^{\mathrm{e}}$ siècle, de nombreux textes officiels et documents ont jalonné l'histoire de la didactique du français ayant pour sujet scolaire l'élève nouvellement arrivé en France, d'abord européen puis issu des flux migratoires d'origine coloniale. Ainsi, c'est le domaine du Français Langue seconde ${ }^{5}$ (FLS), territoire didactique complexe qui s'est peu à peu constitué en phase ou en opposition avec le Français Langue Étrangère (FLE) et le Français Langue Maternelle (FLM). Ce domaine didactique de transition est initialement le produit d'une volonté idéologique et politique de mise en œuvre de dispositifs de prise en charge éducative, destinés à une population scolaire spécifique en relation avec l'histoire de la colonisation française et de l'immigration. (Cuq 1991 ; Vigner 1992)

Le corpus étudié est constitué d'une sélection de quatre circulaires publiées de 1970 à 1990, des Programmes de français au collège publiés de 1996 à 1998, puis en 2008 ; du document d'accompagnement Le français langue seconde paru en 2000 ; du Socle commun (2006); et d'un document de référence publié par le Conseil de l'Europe en 2001 : le Cadre européen commun de référence pour les langues (CECRL). Comment l'aide au développement des compétences scripturales des élèves a-t-elle été envisagée par cette chaîne de prescriptions ministérielles? Comment ces préconisations nationales s'articulent-elles à la perspective européenne globale d'enseignement des langues à l'heure actuelle?

\section{Petite histoire des prescriptions pour l'enseignement de l'écriture aux ENAF}

Dans les années soixante-dix, l'immigration professionnelle s'amplifie suite au développement économique de la France nécessitant un afflux important de main d'œuvre. Les flux migratoires d'origine coloniale se renforcent avec une forte présence de l'immigration algérienne. C'est donc en 1970 et en 1973, que se mettent officiellement en place les classes d'initiation (CLIN) à l'école

\footnotetext{
5 D'après la définition du Dictionnaire de didactique du Français langue étrangère et seconde, le FLS «désigne habituellement un mode d'enseignement et d'apprentissage du français auprès de publics scolaires dont la langue d'origine est autre que le français et qui ont tout ou partie de leur scolarité dans cette langue. Les publics concernés peuvent être des apprenants nouvellement arrivés dans un pays majoritairement francophone (France, Suisse, Belgique, Canada) en vue d'y être scolarisés. Un enseignement spécifique du français leur est dispensé dans des structures d'accueil qui assurent la transition entre une première approche du français comme langue étrangère et le français comme langue de scolarisation »(Cuq 2003 : 109).
} 
primaire, et au collège, les «classes d'adaptation pour enfants de migrants étrangers arrivant en France entre douze et seize ans » (CLAD). La création de ces dispositifs est alors l'objet de directives émanant du Conseil de l'Europe. À cette époque où le monolinguisme domine l'histoire didactique française, les textes programmatiques donnent la priorité à l'objectif de formation linguistique et préconisent l'usage de manuels de méthodes FLE :

La scolarisation des enfants étrangers ne peut s'effectuer dans de bonnes conditions que s'ils acquièrent rapidement l'usage du français, ce qui leur permet de s'intégrer au milieu scolaire et de poursuivre normalement leurs études. Dans tous les cas, l'enseignement de la langue sera dispensé selon les méthodes élaborées pour le français langue étrangère par le Bureau pour l'Enseignement de la Langue et de la Civilisation françaises à l'étranger (BELC) ou par le Centre de Recherche et d'Études pour la Diffusion du Français (CRÉDIF). (Circulaire IX 70-37 du 13-01-1970)

Rappelons qu'à cette époque, domine la méthodologie structuro-globale-audio-visuelle (SGAV) avec des manuels comme Bonjour Line (1963) ou Dialogue Canada (1973). Cette approche ne fait pas de l'écrit une préoccupation première tout au moins au début de l'apprentissage de la langue seconde considérée avant tout comme un moyen de communication orale. La production écrite (Cornaire et Raymond 1994 : 9, 13) est considérée comme un ensemble de sous-savoirs à acquérir selon un modèle hiérarchique en commençant par l'enseignement de règles de grammaire et d'orthographe. L'élève est le plus souvent amené à ne produire que des phrases, d'une manière très guidée et très contraignante. Néanmoins, quelques approches SGAV proposent parfois des petites compositions libres comme exercices d'écriture. Notons que les premières classes d'accueil qui adoptaient cette option méthodologique ont été très contestées car elles se sont rapidement transformées en filières parallèles puis ont marginalisé leur public d'élèves en les orientant vers les sections de l'enseignement spécialisé (SES - SEGPA) pour le secondaire. Cette méthodologie ne peut en effet préparer efficacement les élèves aux écrits travaillés dans les classes ordinaires: narration, description, explication, argumentation.

Après le choc pétrolier de 1973 et la crise économique, l’immigration des travailleurs étrangers diminue. L'enseignement des langues et cultures d'origine (ELCO) est alors officiellement introduit avec des accords et des conventions signées avec huit pays ${ }^{6}$. Les motifs politiques invoqués sont doubles dans la mesure où il s'agit de reconnaître les langues et cultures d'origine pour améliorer l'accueil, permettre une meilleure communication avec la famille, sauvegarder l'identité culturelle d'origine et accompagner la politique du regroupement familial mais aussi d'une manière plus ambiguë de faciliter éventuellement le retour au pays. D’après la sociologue Gabrielle Varro

${ }^{6}$ Portugal (1973), Italie et Tunisie (1974), Espagne et Maroc (1975), Yougoslavie (1977), Turquie (1978) et Algérie (1982). 
(1997), l'idée de prendre en considération les langues d'origine n'est pas nouvelle, et la circulaire suit des directives européennes tout en se référant à un texte de 1939, qui reconduit des dispositions déjà prescrites en 1925, 1927 et 1929 :

Le maintien des enfants étrangers dans la connaissance de leur langue et de leur culture d'origine peut constituer un élément positif de l'adaptation même de ces enfants dans les établissements scolaires français. (Circulaire $n^{\circ} 75-148 d u$ 09-04-1975)

Au plan didactique, une note de la Direction de l'Évaluation du Ministère de l'Éducation Nationale du 25 juin 1975 recommande d'aider les élèves « à maintenir ou à retrouver la pratique de la langue nationale » pour « faciliter chez eux la structuration de la langue française ». Cette précision s’inscrit dans une évolution de la linguistique pragmatique qui dans l'approche dite inter-linguistique analyse les interactions entre langue étrangère et langue maternelle. Elle rejoint aussi les travaux presque contemporains sur l'interdépendance linguistique, menés par le linguiste Jim Cummins (Université de Toronto) qui accorde à la langue d'origine un rôle prépondérant dans l'apprentissage de la langue d'accueil (1979). Cependant, aucune indication n'est donnée dans ces textes quant aux contenus à enseigner et au-delà des bonnes intentions, l'objectif second, en ce début de période de crise économique est de faciliter le retour au pays :

Il est en outre apparu souhaitable tant pour l'adaptation de ces élèves dans les classes françaises qu'en vue de leur retour éventuel dans leur pays de faciliter pour les intéressés le maintien de leur langue et de leur civilisation d'origine. (Circulaire $n^{\circ} 77-345$ du 28 septembre 1977)

Dix ans plus tard, pour lutter contre l'amalgame entre les élèves nouvellement arrivés en France et les enfants en difficulté scolaire ainsi que pour éviter une tendance ségrégative, la circulaire 86-119 du 13 mars 1986 va réaffirmer le caractère temporaire de la scolarisation en classe d'accueil. Pour ce qui concerne l'apprentissage de la langue, le texte reprend à son compte le concept de compétence de communication :

La capacité de communiquer en français est une condition indispensable à l'intégration de l'enfant étranger dans l'école française, à son accès à la formation qu'elle dispense et donc à la réussite scolaire. [...] Ces structures scolarisent de façon temporaire les seuls enfants étrangers non francophones nouvellement arrivés en France.

Ces préconisations publiées en 1986 montrent que l'institution intègre les évolutions de la didactique du FLE des années soixante-dix, accompagnées de la publication de l'ouvrage Un niveau-senil' (Coste 1976), de l'arrivée de nouvelles références en pragmatique, en sociolinguistique, en anthropologie du langage, notamment avec Dell Hymes et le concept de compétence de communication (1984).

\footnotetext{
${ }^{7}$ Instrument de référence pour la description de la langue française, utilisé pour la conception de programmes et de manuels.
} 
Par ailleurs, la circulaire précise aussi les modalités de recrutement et de formation des enseignants : trois ans d'ancienneté au minimum et une formation en didactique du FLE. Précisons que les méthodes contemporaines de français langue étrangère s'appuient sur l'approche communicative (AC) généraliste, sans aucune prise en compte du français langue de scolarisation et des apprentissages ${ }^{8}$. En effet, si l'approche communicative privilégie la communication orale dans de multiples situations dites «authentiques», les activités de production écrite qu'elle propose favorisent les situations de communication de la vie sociale quotidienne, notamment les formes épistolaires, ce qui ne peut faciliter l'acculturation des élèves allophones et l'intégration des formes discursives complexes de la culture scolaire.

En 1995, les nouveaux programmes de français pour le collège mentionnent pour la première fois le Français Langue Seconde. La notion de FLS jusqu'alors réservée à «l'usage du français dans les anciennes colonies en contexte linguistique multilingue, bilingue ou exolingue » (Cuq 1991 : 55) s'étend au contexte scolaire français métropolitain. La référence au FLS est explicite dans le préambule général des programmes et dans tous les documents d'accompagnement pour l'ensemble des niveaux au collège. Sémantiquement, le texte officiel ne désigne plus les élèves du dispositif FLS par la négative (élèves non-francophones) mais introduit le terme allophone, premier indice de la prise en compte de leurs acquis scolaires antérieurs et de leur répertoire linguistique et culturel. Du point de vue didactique les préconisations positionnent clairement l'enseignement du FLS au carrefour de l'enseignement du FLM et de celui du FLE : « contenus et méthodes s'inspirent des didactiques du français langue maternelle et du français langue étrangère » (Ministère de l'Éducation Nationale 1999 : 17).

Le texte prend encore appui sur le niveau-senil et les premiers manuels de l'approche communicatives puisque «les sujets proposés à l'écriture : narrations, descriptions ou explications, sont toujours inscrits dans un contexte pragmatique : identification de l'énonciateur, objectif défini du projet d'écriture » mais il recommande enfin d'initier les élèves « aux écrits évalués par le système scolaire » (Ministère de l'Éducation Nationale 1999 : 31).

Reflétant les avancées des recherches en didactique de l'écrit ${ }^{9}$ et en critique génétique (Grésillon 1994) ainsi que les études sur les brouillons d'écoliers (Fabre 1990), les prescriptions insistent sur les démarches d'aide à la reformulation puisque dès le début de la scolarité dans le

\footnotetext{
${ }^{8}$ Notion popularisée par Michèle Verdelhan-Bourgade (2002).

9 Travaux de recherche en didactique de l'écriture : programmes de l'Institut National de Recherche Pédagogique (INRP) EVA, co-direction Maurice Mas et Claudine Garcia-Debanc (1982-1990) et REV, co-direction Sylvie Plane et Gilbert Turco (1990-1995).
} 
secondaire, «écriture et réécriture d'un brouillon sont systématisées, avec ou sans le professeur» (Ministère de l'Éducation Nationale 1999 : 35). Le brouillon acquiert ainsi un nouveau statut dans la pédagogie de l'écriture.

Puis le document détaille les différentes étapes de l'enseignement de l'écriture en renvoyant sur le mode d'une intertextualité masquée au modèle d'Hayes et Flower (1980) par la mention des étapes du processus d'écriture :

Une place primordiale est donnée aux productions personnelles qui sont écrites quelle que soit la longueur du texte, en plusieurs phases - brouillons, copies, repérages des erreurs, auto-correction, réécriture et parfois mémorisation du texte final. (Ministère de l'Éducation Nationale 1999)

C'est à ce moment de la lecture des programmes que l'on regrette que les directives ne fassent pas allusion au caractère non linéaire des étapes de ce protocole d'écriture (planification, rédaction, révision), imprécision qui risque de rigidifier l'ordonnancement des tâches proposées aux élèves au détriment de la créativité et du développement de pratiques d'écriture singulières. En revanche, prenant en compte des travaux de recherche plus récents sur l'interlangue (Porquier 1984), les auteurs insistent tout particulièrement sur l'intérêt du traitement des erreurs considérées comme les indices de l'évolution de l'interlangue :

Les activités écrites sont très fréquentes, elles comprennent des phases d'autocorrection et de dialogue entre le professeur et l'élève. L'enseignant considère les erreurs comme des témoignages d'un apprentissage en train de se faire, comme les manifestations d'un processus d'interlangue. (Ministère de l'Éducation Nationale 1999)

Les prescriptions ne se prononcent pas sur la description de la langue enseignée, qui est qualifiée de « langue standard ». Un standard qui reste implicite mais qui élimine toute variation.

Ce texte officiel, qui livre des injonctions mais ne donne cependant pas aux enseignants d'indications sur les activités et tâches à proposer aux élèves, va connaittre des développements et des amplifications. À la suite d'une augmentation importante de l'immigration en 1999, Jack Lang, alors ministre de l'Éducation Nationale commande à un Groupe de Travail Disciplinaire (GTD) coordonné par trois didacticiens Alain Viala, Denis Bertrand et Gérard Vigner, une brochure d'accompagnement intitulée «Le français langue seconde ». Ce document d'accompagnement de 44 pages, paru en 2000 et consacré uniquement au FLS, est une première en réponse à un vide curriculaire et programmatique.

Du point de vue de l'enseignement de l'écriture, le texte brosse à grands traits, un ordonnancement de tâches et de contenus d'apprentissage (voir l'appendice) pour que les objectifs de l'institution soient atteints dans ces classes officiellement reconnues comme plurilingues et 
pluriculturelles. Très curieusement, l'ancien modèle de la «rédaction» disparu des Programmes de 1995, resurgit dans ce contexte didactiquement et sociolinguistiquement complexe :

Le professeur aura pour tâche, outre la mise en œuvre des activités du manuel, de bâtir des exercices autres à propos des textes de la méthode et de proposer des activités d'écrit préparant aux évaluations du FLM et des autres disciplines. Les élèves sont sollicités à l'oral sur un sujet qui les motive, ils sont conduits à des reformulations puis à leur écriture. On commence par quelques phrases simples, d'écriture parataxique, pour évoluer vers de courts textes, l'objectif étant la production de « rédactions » correspondant aux codes scolaires. Les thèmes peuvent être choisis en rapport avec ceux des dialogues de la méthode, ainsi qu'avec l'iconographie souvent abondante. Cette pratique permet d'éviter les cloisonnements entre les activités. (Ministère de l'Éducation Nationale 2000 : 17)

Cet opuscule qui s'inscrit institutionnellement dans la conception et la logique des Programmes de français au collège de 1995, s’inspire des documents d'accompagnement pour la classe de troisième, dernier niveau du collège, premier cycle de l'enseignement secondaire (1999) en vue de recommander l'écriture de « rédactions correspondant aux codes scolaires », c'est-à-dire organisées en trois parties comme les textes argumentatifs à produire en troisième ${ }^{10}$ et les textes répondant au «sujet de réflexion» encore en vigueur au Brevet des Collèges ${ }^{11}$ pour les séries technologiques et professionnelles :

L'écriture d'un court texte, présenté et organisé selon les codes de l'exercice « rédaction » du cursus de français langue maternelle, peut prendre place au tout début de l'apprentissage (un mois environ de présence au collège). [...] L'organisation du texte en trois parties est exemplifiée grâce au sujet donné : la demande de renseignement en introduction, la réponse en développement. Une conclusion est élaborée en commun, par exemple, une demande en retour de description ou d'information sur les modes de vie du correspondant. (Ministère de l'Éducation Nationale $2000: 31$ )

Enfermant très artificiellement des écrits fonctionnels dans une organisation tripartite, cette suggestion pédagogique vise à initier dans l'urgence les ÉNAF aux écrits que les auteurs du document jugent encore évalués par le système scolaire. Cette orientation témoigne des difficultés de l'institution scolaire à proposer des moyens d'enseignement favorisant l'intégration des élèves allophones dans le cursus ordinaire.

\footnotetext{
10 «Les textes produits doivent comporter une introduction, un développement et des éléments de conclusion » (M. E. N. 1999 : 156).

${ }^{11}$ Le diplôme national du brevet évalue les connaissances et les compétences acquises à la fin du collège. Il fait une large part au contrôle continu et comporte 3 épreuves écrites à la fin de la troisième. La loi d'orientation de 2005 a donné au brevet une dimension nouvelle: maitriser les connaissances et les compétences du socle commun, une note de vie scolaire, l'attribution de mentions.
} 


\section{De la complexité actuelle du travail enseignant en classe d'accueil}

Ces difficultés rencontrées par l'institution deviennent celle des enseignants qui en tant que fonctionnaires d'État sont dans l'obligation de concevoir des milieux de travail et des outils (pour eux et pour leurs élèves) en fonction des prescriptions. Leur mission se complique lorsque l'institution leur enjoint de certifier le travail de leurs élèves en encourageant la passation de l'épreuve certificative du $\mathrm{DELF}^{12}$ scolaire calée sur les niveaux définis par le Cadre européen commun de référence pour les langues vivantes (2001). D’un côté, le document Le français langue seconde recommande d'enseigner un français de scolarisation, de l'autre, une épreuve certificative en mai-juin, très probablement précédée d'exercices d'entrainement, évalue les compétences communicatives des élèves en français langue étrangère. Cet examen, volontaire et facultatif, exerce une influence sur les pratiques et est encouragé par l'institution: «la délivrance d'un diplôme national notamment de français langue étrangère peut représenter pour ces élèves une puissante motivation et une reconnaissance de leur intégration» (Ministère de l'Éducation Nationale 2005). Notons que les compétences langagières des élèves en production écrite sont évaluées à la suite de la production de deux textes courts (lettre amicale, message) pour inviter, remercier, s'excuser, informer, demander, féliciter... Écrits fonctionnels sociaux qu'ils ne seront plus amenés à réaliser lorsqu'ils intégreront les cours de français de classe ordinaire où la culture scolaire inscrit ses priorités dans la culture lettrée, où l'étude de la langue et de la littérature interagissent constamment tout en valorisant des codes rhétoriques et des formes discursives n'appartenant pas forcément au répertoire des autres cultures scolaires... (cf. les nouveaux programmes de français pour le collège publiés dans le B. O. hors série du 28 août 2008).

\section{Conclusion}

Les préconisations institutionnelles françaises concernant l'enseignement de l'écriture aux élèves allophones ont bénéficié des recherches menées en psycholinguistique, en didactique du français langue étrangère et en didactique du français langue première. Dans le domaine de l'enseignement de l'écriture, elles témoignent encore d'un certain «flou » et oscillent entre l'écrit de la communication ordinaire et l'écrit des disciplines scolaires. Le document ministériel Le français langue seconde a cherché

\footnotetext{
12 Diplôme d'Études de Langue Française.
} 
à clarifier les enjeux et les modalités de l'enseignement du français aux nouveaux arrivants. Publié en 2000, par le Centre national de documentation pédagogique en liaison avec la Direction de l'enseignement scolaire, il mérite d'être revu en raison de l'importance qu'ont prise en dix ans les recherches scientifiques dans le domaine de l'interculturel et du plurilinguisme.

À l'issue de cette étude, on se rend bien compte que, concrètement, l'enseignement de l'écriture en classe d'accueil est actuellement un travail complexe en raison :

1) de l’hétérogénéité maximale du public concerné (âge, langue-culture d'origine, niveau de scolarisation antérieur),

2) d'un besoin d'outils pédagogiques et de méthodes didactiques pour le français langue de scolarisation,

3) d'une ambiguité des prescriptions nationales qui recommandent aux enseignants d'enseigner un français langue de scolarisation tout en leur enjoignant d'utiliser les outils du Conseil de l'Europe conçus pour l'enseignement des langues vivantes étrangères,

4) et aussi d'une insuffisance actuelle des moyens accordés à la formation des enseignants.

La réflexion sur l'enseignement de la langue de l'école et sur son articulation avec les langues de l'immigration poursuit cependant son chemin, notamment au niveau européen, avec les travaux des chercheurs du Conseil de l'Europe qui après l'élaboration du Cadre européen commun de référence pour les langues vivantes, s’intéressent désormais à l'enseignement de la langue de scolarisation, dans la toute récente publication : Un document européen de référence pour les langues de l'éducation? (Coste, Cavalli, Crisan et Van de Ven 2007). À l'origine non prescriptif, le Cadre européen a fortement influencé les programmes nationaux en langues vivantes étrangères. On peut alors se demander quel sera l'avenir de ce document européen de référence pour les langues de l'éducation et son impact sur les prescriptions institutionnelles dans l'Hexagone.

\section{Références bibliographiques}

Bureau des langues d'Ottawa. 1973. Dialogue Canada. Ottawa. Commission de la fonction publique du Canada.

Circulaire $\mathrm{n}^{\circ} 77-345$ du 28 septembre 1977. B. O. $\mathrm{n}^{\circ} 36$ du 03. 10. 1977. Mise en place de cours de langues et de civilisations nationales en dehors des temps scolaires dans les collèges à l'intention des élèves étrangers.

Circulaire IX 70-37 du 13-01-1970. B. O. n5 du 29. 10. 1970. Classes expérimentales pour enfants étrangers. 
Circulaire 73-383 du 25-09-1973. B. O. n³6 du 4. 10. 1973. Scolarisation des enfants étrangers nonfrancophones, arrivant en France, entre 12 et 16 ans.

Circulaire 75-148 du 09-04-1975. B. O. nº1 du 17. 04. 1975. Enseignements de langue nationale à l'intention d'élèves immigrés, dans le cadre du tiers temps des écoles élémentaires.

Circulaire 86-119 du 13-03-1986. B. O. n 13 du 3 avril 1986. Apprentissage du français pour les enfants étrangers nouvellement arrivés en France.

Conseil de l'Europe. 2001. Cadre européen commun de référence pour les langues. Paris : Didier.

Cornaire, Claudette et Raymond Patricia Mary. 1994. La production écrite. Paris : CLE International.

Coste, Daniel. 1976. Un niveau-seuil. Systèmes d'apprentissages des langues vivantes par les adultes. Strasbourg: Conseil de la coopération culturelle du Conseil de l'Europe.

Coste, Daniel, Marisa Cavalli, Alexandru Crisan et Piet-Hein Van de Ven, dir. 2007. Un document européen de référence pour les langues de l'éducation? Division des Politiques Linguistiques, Conseil de l'Europe . http://www.coe.int/t/dg4/linguistic/Source/Prague07_Terminologie_FR. doc

Cummins, James. 1979. Linguistic interdependance and the educational development of bilingual children. Review of Educational Research 40 : 222-251.

Cuq, Jean-Pierre. 1991. Le français langue seconde: Origine d'une notion et implication didactiques. Paris : Hachette.

Cuq, Jean-Pierre, dir. 2003. Dictionnaire de didactique du français langue étrangère et second Paris : CLE International.

Fabre, Claudine. 1990. Les brouillons d'écoliers ou l'entrée dans l'écriture. Grenoble : Céditel.

Ganvenet, Hélène et Pierre Neveu. 1963. Bonjour Line. Paris : Crédif/Didier.

Grésillon, Almuth. 1994. Éléments de critique génétique : Lire les manuscrits modernes. Paris : PUF.

Hayes, John R. et Linda S. Flower. 1980. Identifying the organization of writing process. Dans Cognitive processes of writing, sous la direction de Gregg Lee et Steinberg Erwin, 3-30. Hillsdale, NJ : Lawrence Erlbaum.

Hymes, Dell. 1984. Vers la compétence de communication. Paris : Didier.

Ministère de l'Éducation Nationale.1995. Programmes de français au collège : CNDP.

Ministère de l'Éducation Nationale de la Recherche et de la Technologie. 1999. Programmes et accompagnements. Français. Coll. Enseigner au collège. [1996]

Ministère de l'Éducation Nationale. 1999. Programmes et accompagnements : Français au collège : CNDP.

Ministère de l’Éducation Nationale. 2000. Le français langue seconde. Coll. Collège, Série Repères: CNDP

Ministère de l'Éducation Nationale. 2005. Circulaire n 2005-067 du 15-04-2005. B. O. nº18 du 05. 05. 2005 : préparation de la rentrée 2005.

Ministère de l'Éducation Nationale. 2006. B. O. n²9 du 20 juillet 2006. http://www.education.gouv.fr/bo/2006/29/MENE0601554D. htm 
Ministère de l'Éducation Nationale, de l'enseignement supérieur et de la recherche. 2008. La scolarisation des élèves nouveaux arrivants non francophones au cours de l'année scolaire 2004-2005. Dans Note d'information 2-2008, note d'information de la Division de l'Évaluation et de la Prospective. http://www.education.gouv.fr/stateval

Nonnon, Elisabeth. 1991. Difficultés du langage écrit et oral chez les enfants de l'immigration en échec scolaire : Quelques éléments d'analyse. Enfance 45(4) : 335-354.

Porquier, Rémy. 1984. Communication exolingue et apprentissage des langues. Dans Acquisition d'une langue étrangère III. Paris : Presses Universitaires de Vincennes : 17-47.

Varro, Gabrielle. 1997. Les élèves «étrangers » dans les discours des institutions et des instituteurs. Langage et société $80: 73-99$.

Verdelhan-Bourgade, Michèle. 2002. Le français langue de scolarisation: Pour une didactique réaliste. Paris : PUF.

Vigner, Gérard. 1992. Enseigner le français langue seconde. Paris : CLE International. 


\section{Appendice}

Ministère de l'Éducation Nationale, 2000, Le français langue seconde, Paris, CNDP. Les contenus d'apprentissage (p. 25 \& 27)

\begin{tabular}{|c|c|}
\hline Niveau débutant & Niveau perfectionnement \\
\hline $\begin{array}{l}\text { - Organiser un texte écrit } \\
\text { - Écrire une courte lettre (pour donner de } \\
\text { ses nouvelles, demander un renseignement, } \\
\text { exprimer un désaccord, une plainte) } \\
\text { - Raconter des histoires de son pays } \\
\text { - Rédiger un récit d'expérience personnelle } \\
\text { - Expliquer un événement, une situation } \\
\text { - Justifier un choix, une préférence }\end{array}$ & $\begin{array}{l}\text { - Raconter une histoire vécue ou inventée, selon } \\
\text { une visée particulière (pour faire partager un } \\
\text { sentiment de crainte, une vision amusante, le } \\
\text { caractère émouvant de la situation, pour s'excuser, } \\
\text { se justifier, se plaindre, etc.) } \\
\text { - Rapporter un événement (narrer + expliquer) de } \\
\text { façon objective } \\
\text { - Associer la narration et l'explication } \\
\text { - Réécrire un texte cohérent à partir d'extraits } \\
\text { variés (activités de synthèse) } \\
\text { - Soutenir un point de vue, justifier un choix, } \\
\text { conseiller/déconseiller } \\
\text { - Pratiquer des jeux poétiques } \\
\text { - Prendre des notes, puis convertir des notes en } \\
\text { texte continu } \\
\text { - Reformuler (généraliser, abstraire, exemplifier) } \\
\text { - Transformer une formulation empirique en une } \\
\text { formulation conceptualisée } \\
\text { - Paraphraser, résumer, amplifier }\end{array}$ \\
\hline
\end{tabular}

